\title{
Investigación educativa: la experiencia en el estado de Chihuahua
}

\author{
Evangelina Cervantes Holguín \\ Profesora investigadora \\ Universidad Autónoma de Ciudad Juárez
}

\section{Resumen}

T ste ensayo tiene como propósito recuperar las experiencias de investigación de los académicos e instituciones superiores establecidas en la región. Asimismo, permite conocer el estado que guarda esta actividad intelectual y el desarrollo que ha tenido el campo de la educación en el estado de Chihuahua. De manera concreta se establecen cuatro etapas: antecedentes, primeras experiencias de investigación (1984 a 1990); cambios y desafios (1990 al 2000); crecimiento (2000 al 2009) y apertura y expansión (2009). En esta última fase, se destaca la pertinencia de la REDIECH como espacio para generar, desarrollar, fortalecer y difundir la actividad investigativa en esta entidad.

Palabras clave: investigación, formación, teoría, actitud crítica

\section{Presentación}

La investigación constituye la tarea sustantiva del quehacer científico. Cualquier ciencia requiere de espacios permanentes para revisar sus supuestos, valorar sus principios, identificar ausencias y establecer mecanismos para la generación de conocimiento. En el marco de exigencias del siglo XXI, investigar significa asumir una actitud interrogadora, problematizadora, crítica y abierta ante el mundo.

En el ámbito educativo, la investigación representa un proceso necesario para explicar, interpretar y transformar la realidad; "Sin investigación no hay campo de conocimiento. Hay tradición, rutina, copia, reproducción, dependencia y estatismo" (Imbernón, 2007: 7) Así, esta tarea se presenta como una oportunidad para renovar los saberes y las prácticas educativas. 
En México, la investigación educativa (IE) entendida como "el conjunto de acciones sistemáticas y deliberadas que llevan a la formulación, diseño y producción de nuevos valores, teorías, modelos, sistemas, medios, evaluaciones, procedimientos y pautas de conducta en los procesos educativos" (Latapí, 2002: 14), se realiza desde diferentes escenarios, planos y ángulos revelando una gran diversidad de esfuerzos por explicar y comprender el fenómeno educativo. Si bien, en este ámbito se requiere de procesos de investigación constante, también se necesita que el conocimiento generado sea analizado a fin de valorar su calidad y pertinencia. En este sentido, investigar en y sobre la educación se delinea como una acción previa en el diseño de estrategias tendientes a la mejora de los servicios educativos en cualquier región.

\section{La investigación educativa}

Reconstruir el proceso que ha seguido la IE en la región exige establecer un marco conceptual que oriente su sistematización y análisis. De inicio, conviene delimitar el significado de la IE como quehacer académico a fin de argumentar su consolidación como campo.

Definir conceptualmente la IE es una tarea compleja. No existe una noción absoluta, aplicable a todos los tiempos y contextos. Su significado está determinado por cuestiones de orden epistémico, teleológico, metodológico, entre otras.

En la literatura sobre el campo, es común encontrar conceptos de IE que se posicionan en ángulos opuestos. Desde la visión de Kerlinger (en Imbernón, 2004), investigar en educación, significa elaborar teorías que expliquen los fenómenos educativos. Por su parte, Elliott (en Gimeno y Pérez, 2005), afirma que la investigación recibe el calificativo de educativa cuando pretende no solo ser una investigación sobre educación, sino que educa a través de un proceso de investigación que transforma y perfecciona la práctica.

A fin de superar la distancia entre la teoría y la práctica, algunos autores asumen la IE bajo conceptualizaciones más inclusivas, recuperando el sentido constructivo de esta actividad. De esta forma, la IE "se puede conceptuar como un nuevo conocimiento sobre lo educativo [...] que además genera el ejercicio de una práctica distinta en la producción del conocimiento" (Gutiérrez, en Piña y Pontón, 2002: 28).

Desde un enfoque teleológico, un sector de especialistas de la IE toman en cuenta los fines de esta tarea como referente para su conceptualización. De manera general, destacan dos posiciones, por ejemplo, Ducoing (en Martínez, 2010: 6) sostiene que "la finalidad de cualquier investigación no es la formulación de propuestas, recomendaciones, diseños, etc., sino primordialmente comprender la realidad educativa”. Así, se privilegia la orientación del trabajo heurístico a objetivos comprensivos explicativos como una acción distinta, e incluso lejana, de la intervención educativa. Con respecto a la segunda posición, Weiss (2003: 36) define a la IE como aquella conformada por estudios, en forma de intervenciones educativas, diagnósticos, sistematizaciones de experiencias, evaluaciones y proyectos, que contribuyen a racionalizar la acción educativa. Desde esta perspectiva, la investigación promueve momentos para la intervención, transformación y mejora de la realidad educativa. En este senti- 
do, Latapí (2002: 14) afirma que la IE se relaciona con la innovación educativa, entendida como "el conjunto de actividades intencionales y organizadas, orientadas a implantar los resultados de la IE, con el fin de mejorar los procesos y sistemas educativos".

En el presente trabajo se adopta la perspectiva señalada por Weiss, al considerar a la IE como una serie de actividades, con diversos alcances, orientadas a la explicación, comprensión y transformación de una realidad compleja que requiere lecturas, individuales y colectivas, pluridisciplinarias, interparadigmáticas y multirreferenciales, tendientes a la producción de conocimiento científico sobre la educación.

\section{La investigación educativa en el estado de Chihuahua}

La investigación constituye una tarea prioritaria en el camino a la producción permanente del conocimiento. Una sociedad informada posee elementos que le permiten decidir oportunamente sobre las acciones que, traducidas en políticas públicas coherentes, garantizan a sus miembros mejores niveles en su calidad de vida.

Adentrarse en los esfuerzos de los docentes chihuahuenses por fortalecer la IE, exige revisar los antecedentes que contribuyeron a la conformación de este quehacer científico. La primera etapa de la IE en la entidad, que se ha denominada antecedentes, se puede ubicar a partir del año 1984, cuando los estudios de educación normal se elevan al nivel de licenciatura (SEP, 2003). Las primeras experiencias de investigación educativa "coinciden con el establecimiento del nivel de licenciatura que ubicó a las escuelas normales como instituciones de educación superior y consecuentemente la investigación se constituyó en una de las funciones sustantivas" (Franco, 2009: 19).

En la actualidad, los planes de estudio de las normales pretenden desarrollar en el estudiantado diversas competencias entre las que destaca la disposición y capacidades propicias para la investigación científica, como la curiosidad, capacidad de observación, método para plantear preguntas y para poner a prueba respuestas y reflexión crítica con el propósito de mejorar los resultados de la labor educativa (DGESPE, 1999).

En esta década destacan los esfuerzos de la Comisión Estatal para la Planeación de la Educación Superior al organizar encuentros de investigación. A finales de los años 80 , se realiza el primer encuentro "en donde las instituciones de formación de docentes participaron tanto en su organización, como en las mesas de trabajo presentando ponencias producto de las investigaciones realizadas, lo que contribuyó a difundir los resultados, pero sobre todo a retroalimentar su quehacer" (Franco, 2009: 20).

En el camino hacia el fortalecimiento de la IE en el estado, la década de los años 90 representó un escenario de Cambios y desafíos, que configura lo que se ha denominado la segunda etapa de la IE en la región. Entre las dificultades puede señalarse la presencia de académicos con ciertas limitantes en su formación y experiencia para la investigación, recursos insuficientes $\mathrm{y}$ apoyos institucionales y gubernamentales incipientes. En esta época se establecen las primeras experiencias de superación profesional para el magisterio, que en forma de maestrías en educación brindaban una formación primaria para la investigación. 
Como resultado de la realización de diversas acciones ante las instancias oficiales y sindicales, se crearon programas de maestría en la Universidad Pedagógica Nacional (UPN), en el Centro Chihuahuense de Estudios de Posgrado (CCHEP) y el Centro de Investigación y Docencia (CID), conformándose como los espacios de Superación Profesional con mayor relevancia entre el magisterio local. Aún cuando los posgrados en la entidad tenían el carácter profesionalizante, consideraban en sus programas cursos con tendencia a la investigación lo que permitía al estudiantado desarrollar habilidades para tal tarea.

En la entidad, se han elaborado diversos estudios sobre la educación, lo que ha contribuido a la conformación de equipos de trabajo y el desarrollo de competencias institucionales e individuales para la investigación. En el año 1994, se realiza el Proyecto Estatal de la Calidad Educativa de los Servicios Educativos del Estado de Chihuahua (SEECH), acción derivada del programa de Innovación Educativa: Calidad Total en la Educación (SEC, 2003).

A mediados de los años 90, se encomendó a la Coordinación de Investigación y Desarrollo Académico (CIyDA), de la Dirección General de Educación y Cultura del Gobierno del Estado, la elaboración de la Agenda educativa de la administración 1992-1998 con la finalidad de reestructurar la administración pública del gobierno estatal para mejorar la educación en cuanto a sus metas como bien público: cobertura, eficiencia, calidad, equidad y pertinencia (Heurística Educativa, 1998).

De enero de 1994 a junio de 1995 se realizó el Diagnóstico de la Educación Primaria en el Estado de Chihuahua, en donde se presentaba un panorama general de los servicios educativos en el subsistema federalizado, del cual surgieron propuestas de investigación individuales apoyadas por la SEP-ConACYT (SEC, 2003).

En el año de 1995, el CID realizó dos investigaciones: Atención a los niños de los cruceros $\mathrm{y}$, Los requerimientos de educación preescolar y primaria en la población indígena migrante, ambas bajo el convenio entre el Gobierno del Estado y la Sección 8 del Sindicato Nacional de Trabajadores de la Educación (SNTE). Durante el período de 1996-1997 esta institución firmó otro convenio con el Instituto Nacional de Antropología e Historia que hizo posible la realización del Diagnóstico de la Educación Primaria en el Municipio de Allende (Franco, 2009).

En 1999, se realiza el trabajo de investigación titulado La supervisión escolar de la educación primaria en México. Prácticas, desafíos y reformas, que integraba el esfuerzo de varias Instituciones de Educación Superior (IES) y el apoyo de la UNESCO, a través del Instituto Internacional de Planeamiento de la Educación (IIPE) con sede en París (Calvo, 2002).

En el marco de la tercera etapa, denominada de crecimiento, a partir del año 2001, Heurística Educativa trabaja en el análisis pedagógico desde el Programa Escuelas de Calidad (PEC), que nace como una iniciativa de reforma de la gestión institucional y escolar con la finalidad de superar diversos obstáculos para el logro educativo. En él participan los profesores de las escuelas públicas de educación básica, en todos sus niveles y modalidades (SEB, s.f.). Esta experiencia ha permitido identificar líneas de investigación tendientes a analizar la práctica de los docentes en Chihuahua, los logros académicos y eficacia social de las escuelas, las perspectivas 
de los alumnos sobre las escuelas PEC, el análisis de la práctica pedagógica videograbada, entre otras.

Una de las necesidades que actualmente existen en el rubro educativo es el desarrollo de investigación que permita valorar la producción existente, con el fin de determinar oportunidades para la generación de nuevos conocimientos. En esta línea, un avance significativo en la IE local lo constituye la elaboración de los Estados de conocimiento de la IE en el estado de Chihuahua, proyecto coordinado por el Departamento de Investigación Educativa de la Secretaría de Educación y Cultura (SEC) bajo el esquema de fondos mixtos del ConACYT con la participación entusiasta de investigadores y académicos chihuahuenses (CONACYT, 2006). La realización de perspectivas estatales de los estados de conocimiento se planteó por el CoMIE desde el año 2001, al convocar, tanto a las instituciones de posgrado como a los investigadores educativos, a colaborar en el esfuerzo de construir los estados de conocimiento en las entidades federativas con el objetivo de abrir espacios de reflexión e intercambio de ideas sobre la actividad educativa para enriquecer y consolidar la investigación (Ramos, 2009).

En el año 2006, se efectúa el II Encuentro de Investigación Educativa permitiendo el intercambio académico entre docentes, investigadores, alumnos de instituciones de posgrado, e integrantes de la comunidad educativa en la presentación de ponencias, producto de protocolos dictaminados para fines de tesis, avances y resultados de investigaciones terminadas (SEC-SEECH, 2006).

En este mismo año, se forma un consejo editorial colaborativo entre las instituciones de formación y actualización docente de la entidad, con la responsabilidad de publicar la revista Ecos de la educación. Este espacio constituye una oportunidad para la difusión de los trabajos de IE producidos en la entidad.

Pese a los avances en la consolidación de la IE en la región, para Ordóñez (2006), las actividades de investigación han sido insuficientes, debido a la escasez de proyectos, principalmente porque la mayoría de las Instituciones de Educación Superior (IES) habían priorizado a la docencia sobre la investigación dentro de sus actividades sustantivas. Además, las instituciones de posgrado orientados a la formación en investigación eran limitadas.

No obstante, en el año 2008 se realizó el III Encuentro de Investigación Educativa con el propósito de promover el diálogo entre las personas interesadas en la realización de investigaciones en el sector educativo. Entre las actividades, se presentaron, discutieron y revisaron los resultados de trabajos sobre sujetos de la educación; políticas educativas; estudios con perspectiva de género; procesos de formación y prácticas educativas con la meta de buscar y proponer alternativas para mejorar la calidad educativa en Chihuahua (El ágora, 2008).

En este mismo año, la Dirección de Educación Primaria y el Programa de Investigación y Evaluación de los SEECH convoca al I Encuentro regional de investigación educativa con el objetivo de crear un espacio de encuentro que promueva el diálogo entre los docentes, investigadores y otros actores de la educación; presentar, analizar y valorar la IE que se realiza en las escuelas primarias del estado, identificando problemas y necesidades de la práctica educativa; promover la formación, actualización 
y superación de los actores de la educación, facilitando el acceso a los productos de la investigación, así como impulsar la IE como una herramienta que le permite al docente estudiar y reflexionar sobre su práctica (SEC, 2008).

A partir del 2009, se gesta la cuarta etapa denominada de apertura y expansión, considerando la realización de investigaciones interinstitucionales, foros y encuentros, así como la publicitación en Internet de la agenda estatal de IE con acceso a través del catálogo a 448 fichas (SEC, 2009).

En este año, el Poder Ejecutivo Estatal a través de la SEC y el CoNACYT organizaron el Primer Foro Estatal de Resultados en Ciencia y Tecnología. FOMIX 2009 Chihuahua, con el objeto de difundir los resultados de investigaciones apoyadas por el Fondo Mixto ConacytGobierno del Estado, además de propiciar el encuentro entre los actores involucrados en el desarrollo científico, tecnológico, económico y social de la Entidad.

\section{4: Antecedentes}

Primeras experiencias de investigación Formación inicial
Hasta la fecha, el fondo apoya más de 160 proyectos de investigación científica, tecnológica y de innovación. En el área III Educación, se reportan 26 trabajos con un monto de apoyo de 5 millones de pesos (SEC, 2009). Entre los cuales destaca la presentación de los avances en la elaboración de los Estados de conocimiento de la IE en Chihuahua.

En este año, profesores, académicos de las unidades UPN en Chihuahua y el equipo de Heurística Educativa (HE) colaboraron en la investigación Estándares para la educación básica a fin de impulsar procesos de mejora académica continua en todas las escuelas de México (SEB, 2009).

Durante los primeros meses del año 2010, se efectuó el IV Encuentro de Investigación Educativa 2010 bajo la coordinación del CCHEP, el CID y la UPN con el fin de contribuir en la búsqueda de alternativas que coadyuven a mejorar la calidad de la educación, a través del intercambio y presentación de los proyectos y productos de la investigación generados en el

1990: Cambios y Desafíos

Superación profesional

Formación para la investigación

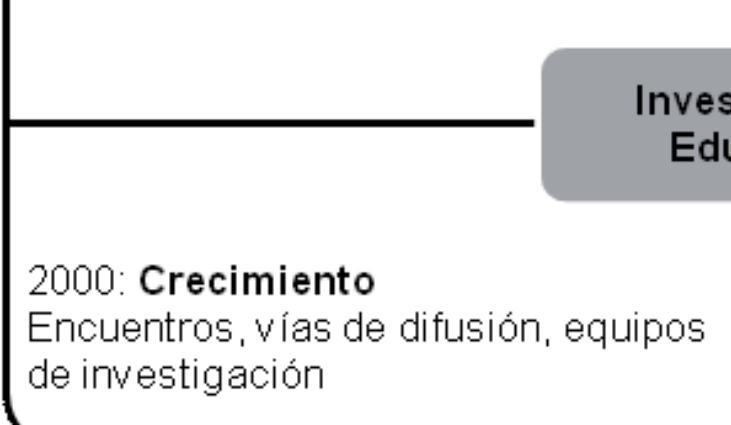

2009: Apertura y expansión Investigación interinstitucional, foros, catálogoweb

Cuadro No. 1. Etapas de la Investigación Educativa en Chihuahua 
estado, en torno a la realidad educativa, nacional y regional (SEC, 2010).

Además, para dar continuidad al trabajo de difusión y promoción de la IE generada en la entidad, recientemente se formalizó la Red de Investigadores Educativos Chihuahua como una organización horizontal de investigadores educativos del estado, orientada a la generación, desarrollo, fortalecimiento y difusión de la actividad investigativa de la entidad. Entre sus principales objetivos se encuentra realizar investigación en el campo educativo; promover la creación de redes y grupos de trabajo; promover acciones tendientes a la formación permanente para la investigación; promover y organizar eventos y encuentros académicos; establecer relaciones con otros organismos y asociaciones científicas o académicas, entre otros (Rediech, 2010).

\section{Comentarios finales}

La investigación educativa en Chihuahua puede valorarse como una acción prioritaria. Investigar el fenómeno educativo ofrece la posibilidad de conocer las realidades que convergen en el ámbito del aula, la escuela y el sistema educativo en general; evaluarlas, describirlas e interpretarlas a fin de diseñar estrategias para su transformación.

La exposición anterior, refleja avances significativos en la consolidación de la IE en la región lo que se manifiesta en la conformación de redes interinstitucionales, la formación de investigadores educativos, el establecimiento de canales para la difusión y publicación de los resultados de investigación, así como el apoyo a los centros de posgrado en educación.
Sin embargo, hacer investigación siempre será un reto, una tarea inacabada, en permanente desarrollo, colectiva, compleja e indispensable para cualquier sociedad en búsqueda de la equidad y justicia social. Además, analizar lo investigado permite develar nuevas rutas de búsqueda, diálogo y construcción en torno a la educación, para lo cual es conveniente apoyar la IE en el estado, fortalecer las instituciones de posgrado en educación, garantizar las condiciones para la elaboración de investigación individual y colectiva, así como, replantear la actualización permanente de los investigadores.

Entre los retos de la IE en la región, se vislumbra redimensionar la realización de diagnósticos, que permitan conocer los requerimientos y necesidades de la actividad educativa para enfrentar con éxito la diversidad cultural y geográfica, integrar en los referentes teóricos el enfoque andragógico y la perspectiva de género, analizar la conformación de redes y comunidades de aprendizaje, de valorar las experiencias de investigación colectiva, así como la formación para la investigación.

\section{Bibliografía}

Calvo, B. (2002). La supervisión escolar de la educación primaria en México: Prácticas, desafíos y reformas. Paris: UNESCO.

CONACYT (2006). Convocatoria 2006-01. Recuperado el 27 de agosto de 2009, de Fondo Mixto CONACYT-Gobierno del Estado de Chihuahua: http://www.conacyt.mx/fondos/Mixtos/Chihuahua/2006-01/Chihuahua_Resultados-Pertinencia_2006-01.pdf

DGESPE (1999). Los rasgos deseables del nuevo maestro: Perfil de egreso. Recuperado el 28 de agosto de 2009, de Plan de Estudios, Licenciatura en Educación Preescolar: http://normalista. ilce.edu.mx/normalista/index.htm

El ágora (2008). III Encuentro de Investigación Educativa. Recuperado el 27 de agosto de 2009, de El ágora. Periódico digital: http:// www.elagoradechihuahua.com/III-Encuentro-de-Investigacion, 5172.html

Franco, M. C. (2009). La investigación educativa en Chihuahua. Recuento 1985-1999. Recuperado el 27 de agosto de 2009, de Centro de Investigación y Docencia: http://www.cid.edu.mx/

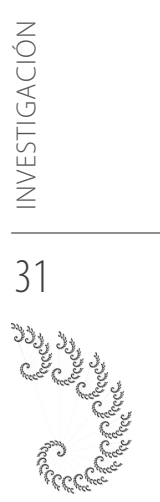


images/pdfmaster/PDFweb/Pag19-25x.pdf

Gimeno, J., y Pérez, A. (2005). Comprender y transformar la enseñan$z a 11^{a}$ Edición ed. España: Ediciones Morata.

Heurística Educativa (1998). Fijan chihuahuenses sus bases educativas. Recuperado el 28 de agosto de 2009, de Foro 21: http://he.heuristicaeducativa.org/NewForo21x/ FORO21/11edicion/02.htm

Imbernón, F. (2007). 10 ideas clave: La formación permanente del profesorado: Nuevas ideas para formar en la innovación y el cambio. Barcelona: Editorial GRAO.

Latapí, P. (2002). La investigación educativa en México. México: Fondo de Cultura Económica.

Martínez, R. (2010). Estado de conocimiento del campo de la investigación educativa. Recuperado el 23 de enero de 2010. Departamento de Investigación Educativa de SEC. http://chihuahua. gob.mx/atach2/investigacioneducativa/uploads/Invconcept. pdf

Ordóñez, G. M. (2006). Los retos de la politica social en la frontera norte de México. México: COLEF/Plaza y Valdés.

Piña, J. M., y Pontón, C. B. (2002). Cultura y procesos educativos. México, D.F.: Plaza y Valdés.

Ramos, J. E. (Enero de 2009). La investigación educativa en el norte de México. Recuperado el 27 de Agosto de 2009, de Revista Electrónica de Investigación Educativa Sonorense: http://www. redies.org/REDIES2/ART_6_REDIES_2.pdf

REDIECH (19 de Abril de 2010). Proyecto de la REDIECH. Chihuahua, Chih, México.

SEB (s.f.). Descripción del Programa. Recuperado el 28 de Agosto de 2009, de Programa Escuelas de Calidad: http://basica.sep.gob. $\mathrm{mx} /$ pec/start.php?act $=$ programa\&sec $=$ des
SEB. (2009). Referentes para la educación básica en México . Recuperado el 28 de agosto de 2009, de Estándares para la educación básica: http://referenteseducativos.net/

SEC (2010). Encuentro de Investigación Educativa 2010. Recuperado el 10 de junio de 2010, de Universidad Pedagógica Nacional Unidad 081: http://www.upn081.edu.mx/data/files/zip/ConvocaEnc2010final.pdf

SEC (2009). Estado del Conocimiento de la Investigación Educativa en el Estado de Chihuahua. Recuperado el 19 de diciembre de 2009, de Departamento de Investigación: http://www.chihuahua.gob.mx/investigacioneducativa/

SEC (2003). Hacia una politica integral para la formación y el desarrollo profesional de los maestros. Diagnóstico del Sistema Estatal de Formación Docente: Chihuahua. Chih.

SEC (2008). I Encuentro regional de Investigación Educativa. Convocatoria. Recuperado el 27 de agosto de 2009, de Coordinación Estatal de Tecnología Educativa: http://www.cetechihuahua.gob.mx/portal/export/sites/default/dep/descargas/ Convocatoria_I_Encuentro_Regional_de_Investigacion_Educativa.pdf

SEC (2009). Primer Foro Estatal de Resultados en Ciencia y Tecnología. Convocatoria. Recuperado el 30 de agosto de 2009, de Secretaría de Educación y Cultura: http://www.chihuahua.gob. $\mathrm{mx} / \mathrm{sec} /$ Plantilla5.asp?cve_Noticia $=5433 \&$ Portal=sec

SEC-SEECH (2006). Memoria electrónica del II Encuentro de investigación educativa. Chihuahua, Chih., México.

SEP (2003). El mejoramiento de la gestión institucional en las escuelas normales. México.

Weiss, E. (2003). El campo de la Investigación Educativa. México: Grupo Ideograma Editores. 